\title{
Surgical Management on Posterior Uveitis with Vitreous Opacity Suspected Retinal Detachment
}

\author{
Firda Ayu Muthie ${ }^{1}$, Sauli Ari Widjaja ${ }^{1,2}$, Wimbo Sasono ${ }^{1,2}$, Muhammad Firmansjah ${ }^{1,2}$, Ima Yustiarini ${ }^{1,2}$, \\ Ady Dwi Prakosa ${ }^{1,2}$, Moestidjab ${ }^{1,2}$, Gatut Suhendro ${ }^{1,2}$ \\ ${ }^{1}$ Department of Ophthalmology, Faculty of Medicine Universitas Airlangga, Dr. Soetomo General Academic \\ Hospital Surabaya, Indonesia \\ 2 Vitreoretinal Division, Department of Ophthalmology, Faculty of Medicine Universitas Airlangga, Dr. \\ Soetomo General Academic Hospital Surabaya, Indonesia
}

\begin{abstract}
Introduction: Posterior uveitis entities are varying between infective or non-infective in aetiology. It can affect the adjacent structures such as retina, vitreous, optic nerve head and retinal blood vessels. Vitreous opacity is the most common features of posterior uveitis and posterior segment evaluation is critical to determine the aetiology and management.
\end{abstract}

Case presentation: 32-year-old male with gradual visual loss on right eye since 2 years ago and getting worse 1.5 months before admission. Visual acuity (VA) was hand movement. Posterior segment evaluation revealed vitreous opacity and ultrasound examination showed membrane shaped lesion attached to the optic nerve suggested retinal detachment.

Discussion: Vitrectomy was done for diagnostic and therapeutic purpose. VA was remarkably improved by 6/6 and persist until 6 months post vitrectomy, and progressive improvement on posterior segment.

Conclusion: Surgical in the management of posterior uveitis can be divided based on indication, either for therapeutic or diagnostic purposes or to manage its complications. Vitrectomy is one of the modality to manage vitreoretinal complications associated with uveitis.

Keywords: posterior uveitis, retinal detachment, vitreous opacity, vitrectomy

Cite This Article: MUTHIE, Firda Ayu. SURGICAL MANAGEMENT ON POSTERIOR UVEITIS WITH VITREOUS OPACITY SUSPECTED RETINAL DETACHMENT. International Journal of Retina, [S.I.], v. 3, n. 1, feb. 2020. ISSN 2614-8536. Available at: https://www.ijretina.com/index.php/ijretina/article/view/83 Date accessed: 17 feb. 2020. doi: https://doi.org/10.35479/ijretina.2020.vol003.iss001.83

*Correspondence to:

Firda Ayu Muthie,

Department of Ophthalmology,

Faculty of Medicine Universitas

Airlangga,

Surabaya, Indonesia

firda.muthie@gmail.com

\section{INTRODUCTION}

Posterior uveitis may be focal, multifocal, or diffuse with involvement of posterior segment structures such as choroid, retina, retinal blood vessels and optic nerve head. ${ }^{1}$ The entities are varying between infective or non-infective in etiology. A thorough diagnostic work-up directed by the history of presenting complaints, patient's symptoms and signs, and clinical examination is mandatory. ${ }^{2}$ Pars plana vitrectomy has long been used for the management of various forms of uveitis and allows detailed fundus observation during surgery. 3,4 


\section{CASE PRESENTATION}

A case report of 32-year-old male with chief complain of gradually blurred vision on his right eye since 2 years ago and getting worse 1.5 months before admission. Visual acuity was $1 / 300$ and $6 / 6$ on his right and left eye respectively. Intraocular pressure was perfectly normal on both eyes. He has unremarkable medical history and no history of drug usage. The anterior segment was completely between normal limit on both eyes. No flare or cell were found on both eyes. Posterior segment on his right eye was difficult to be evaluated because of the vitreous opacity.

Ultrasound examination was performed to determine the haziness. B-scan ultrasonography on the right eye showed membrane shaped lesion attached to optic nerve, less mobile with reflectivity around $20-30 \%$ retina choroid sclera (RCS) complex on vitreous cavity.

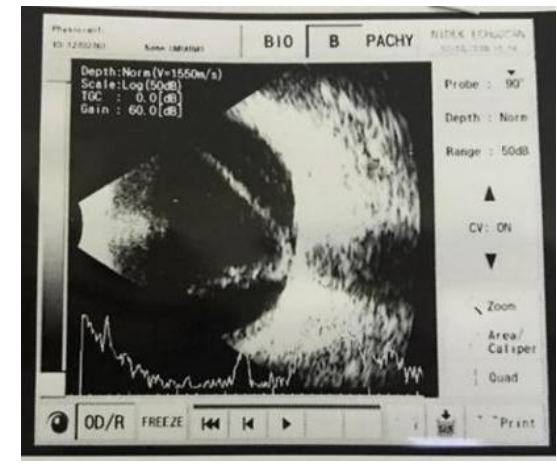

Figure 1. Ultrasound examination on the right eye showed membrane shaped lesion attached to the optic nerve.

Laboratory result was between normal limit but we didn't perform any immunology examination due to resources limitation. Steroid was given orally and topically only after the surgery because there were no sign of inflammation preoperatively. Methylprednisolone $8 \mathrm{mg}$ tapering off was given three times daily orally for about 2 weeks and fluorometholone eye drop was given for about a month on right eye

\section{RESULTS}

Vitrectomy was performed on right eye. There was dense vitreous and membranes in vitreous cavity due to inflammation. Retina was attached. There were diffuse vascular involvement and some retinal bleeding around the optic nerve, but there were no active bleeding nor blood in vitreous was found. Vasculitis was found by the presence of perivascular sheathing and some exudation around the affected vessels. Venous dilatation were found with focal arterial sclerotic. Unfortunately we could not performed vitreous biopsy to this patient due to insurance issues and the patient refused to do this procedure.

Visual acuity on right eye was $1 / 300$ preoperatively and still remain the same in one day post-operatively. On the next day there were significant improvement on right eye by $5 / 12$ and continue to progress by $5 / 8.5$ in a week postoperative followed by a remarkable improvement of posterior segment condition. Two weeks postoperative his visual acuity became 5/5. 6 months follow up post-operatively, the visual acuity remains 5/5. Intra ocular pressure were also monitored for each follow up and it vary between $12.2 \mathrm{mmHg}$ to $17.2 \mathrm{mmHg}$.

Fundus photography had been performed each time the patient came to follow up.

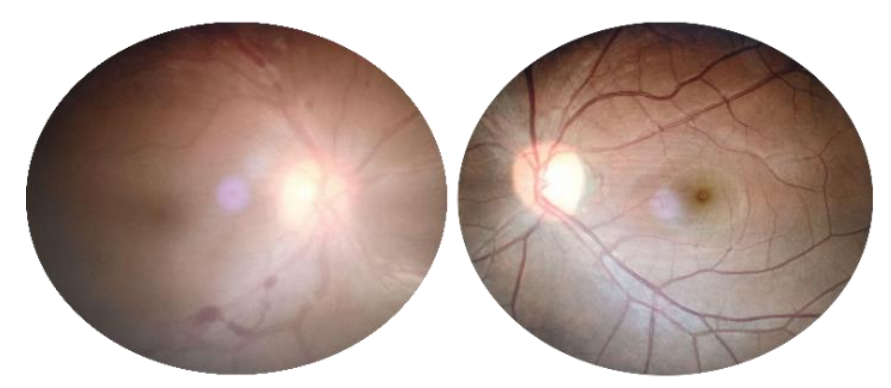

Figure 2. Fundus photography on both eyes 2 days postoperative 


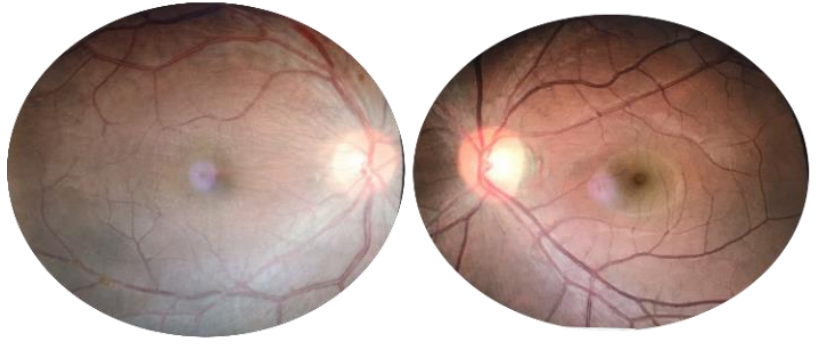

Figure 3. Fundus photography on both eyes a month post-operative

Optical Coherence Tomography Angiography (OCT-A) and Spectral Domain OCT (SD-OCT) was performed 30 days post-operative, and both showed normal configuration. The image showed by angiography was superficial inner retina that contains a vascular projection of retinal nerve fiber layer (RNFL) and ganglion cell layer (GCL). There were no avascular zone nor neovascularization.

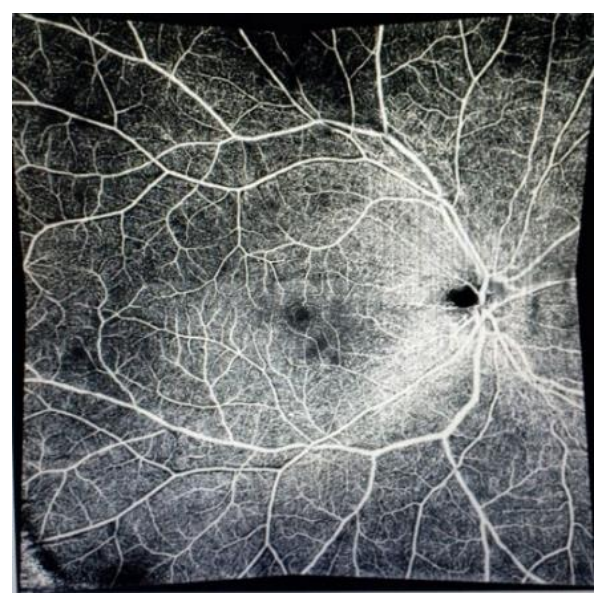

Figure 4. OCT angiography of the right eye showed normal superficial retina vasculature

The SD-OCT showed normal configuration of each retinal layer and there were no membrane was found.

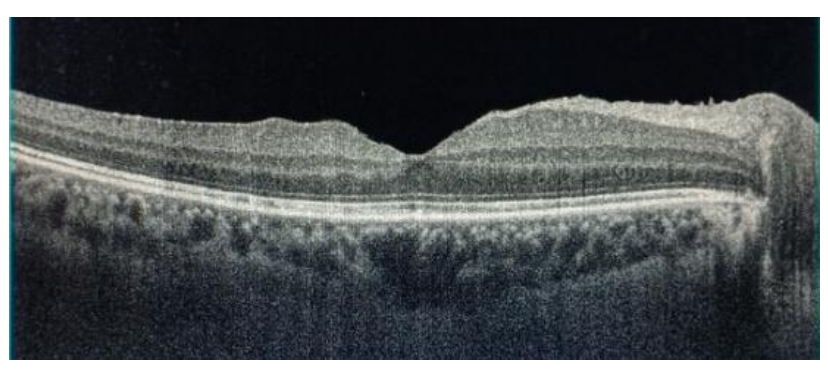

Figure 5. SD-OCT of the right eye 30 days post operative treatment. It shows normal retinal layer and no membrane was found.

\section{DISCUSSION}

The clinical symptoms of the patient was not fully developed as uveitis. But the visual loss happened gradually and became worst and this patient had a good respond to steroid treatment. The ultrasound examination supported the diagnosis of uveitis because of the membrane shape like was found.

Posterior uveitis has a broad differential diagnosis. Once the diagnosis of posterior uveitis is confirmed, next important step is to determine the extent of involvement of the inflammation. ${ }^{1}$

Although epiretinal membrane (ERM) is common in uveitis, ${ }^{5}$ the membrane that seen on ultrasound was only found in vitreous cavity due to the dense opacity of the vitreous and not involving the retina. Because of the dense vitreous opacity in this case, we could not performed OCT. OCT is helpful in confirming the presence of epiretinal membranes and in distinguishing inflammatory macular edema from that due to vitreomacular traction, thereby identifying cases that are more likely to respond to surgical intervention. Epiretinal membranes that are associated specifically with uveitis seem to differ from idiopathic ERM in cellular composition, suggesting that they may emerge through a different pathogenic mechanism. In this case, SDOCT showed normal retinal layer on right eye. ${ }^{6,7}$

The indications for surgery in the management of uveitis can be divided as: ${ }^{5}$

1. Visual rehabilitation: Surgery for removal of cataract, band keratopathy, corneal scars, pupillary membranes, removal of dense vitreous membranes.

2. Management of complications: Anti-glaucoma surgery, vitreous haemorrhage, retinal detachment and chronic hypotony.

3. Diagnostic: Aqueous tap, vitreous biopsy, tissue biopsy (iris, choroid). 
Vitrectomy is an option for atypical clinical presentations of uveitis. It also done for uveitis that not responds to empirical treatment with corticosteroids/ immunosuppressants and for rapidly progressive disease with inconclusive noninvasive work-up or if there is strong suspicion of malignancy. ${ }^{8}$

The vitrectomy technique is using standard threeport vitrectomy because it is preferable while performing combined diagnostic and therapeutic vitrectomy to get the best visualization of the fundus. This method is also allowing better diffusion of intraocular medications and yielding more vitreous sample for analyzation. ${ }^{7}$

In uveitis, the dictum is to operate in a quiet eye, especially, if it is for an elective surgery as for visual rehabilitation. It is mandatory to wait for at least 3 months after the last episode of active disease. ${ }^{8}$ Preoperative ancillary assessment might include Bscan ultrasonography or ultrasonic biomicroscopy in the presence of media opacity or hypotony to detect underlying chorioretinal pathology, such as exudative retinal or choroidal detachment, and cyclitic membranes, which might influence the surgical plan. ${ }^{7}$

\section{CONCLUSION}

Surgery in the management of uveitis can be divided based on indication, either for therapeutic or can be for diagnostic purposes or to manage complications. ${ }^{7}$ Vitrectomy is a good modality to manage vitreoretinal complications associated with uveitis, in this case vitrectomy was useful for diagnostic procedure and for visual rehabilitation. Pre-operative factors include proper patient selection and counseling and pre-operative control of inflammation.
It is now well-recognized that chronic inflammation, even low grade, can irreversibly damage the retina and optic nerve and therefore inflammatory control both pre- and postoperatively is vital. $^{6}$

\section{REFERENCES}

1. Sharma, Preeti, \& Majumder, Parthopratim. (2015). Diagnosis and management of posterior uveitis. Current Indian Eye Research. 2017, 1-10.

2. Biswas, J., Sudharshan, S., \& Ganesh, S. (2010). Current approach in the diagnosis and management of posterior uveitis. Indian Journal of Ophthalmology, 58(1), 29.

3. Kaza, H., Modi, R., Rana, R., Panda, K. G., Barik, M. R., Ali, M. H., \& Basu, S. (2018). Effect of Adjunctive Pars Plana Vitrectomy on Focal Posterior Segment Inflammation: A Case-Control Study in Tuberculosis-Associated Uveitis. American Academy of Ophthalmology 2018:1-7.

4. Sato, T., Kinoshita, R., Taguchi, M., Sugita, S., Kaburaki, T., Sakurai, Y., \& Takeuchi, M. (2018). Assessment of diagnostic and therapeutic vitrectomy for vitreous opacity associated with uveitis with various etiologies. Medicine, 97(2), e9491.3. 15.

5. Kempen JH, Altaweel MM, Holbrook JT, et al. The multicentre uveitis steroid treatment trial: rationale, design, and baseline characteristics. Am J Ophthalmol 2010;149:550-561.

6. Nicholson, B. P., Zhou, M., Rostamizadeh, M. Mehta, P., Agrón, E., Wong, W., Sen, H. N. (2014). Epidemiology of Epiretinal Membrane in a Large Cohort of Patients with Uveitis. Ophthalmology, 121(12), 2393-2398.

7. Vitale, Albert. (2006). Vitrectomy in Patients With Uveitis. Review of opthlamology. Accessed on 22nd 2019: https://www.reviewofophthalmology.com/articl e/vitrectomy-in-patients-with-uveitis 
8. Murthy, S., Pappuru, R., Sangwan, V., Kamat, S., \& Latha, Km. (2013). Surgical management in patient with uveitis. Indian Journal of

(c) (i) $\odot$

This work licensed under Creative Commons Attribution Ophthalmology, 61(6), 284. 